\title{
Density-functional calculations of Esterel twinning in quartz
}

\author{
Bernard Delley and Hans Grimmer \\ Paul Scherrer Institut, CH-5232 Villigen PSI, Switzerland \\ (Received 9 July 2007; published 12 December 2007)
}

\begin{abstract}
Models for Esterel twin interfaces with mirror and rotation symmetry are presented that satisfy stoichiometry at the composition plane. The starting points are unrelaxed models constructed from bulk quartz crystal and keeping the bulk volume per formula unit. The proposed unrelaxed models also maintain the fourfold coordination of all $\mathrm{Si}$ atoms and the twofold coordination of the $\mathrm{O}$ atoms, albeit with significant variation of bond length and bond angles at the composition plane. More realistic atomic positions were sought with density functional calculations. Relaxation energies are quite significant, as the initial unrelaxed interface energy is about $15 \mathrm{eV}$ above the relaxed energy for our cells containing two twin interfaces. After relaxation, bond length and bond angles are close to bulk quartz values. It was found that reflection twins have lower interface energy than rotation twins.
\end{abstract}

DOI: 10.1103/PhysRevB.76.224106

PACS number(s): 61.72.Mm, 71.15.Nc

\section{INTRODUCTION}

The stable modification of $\mathrm{SiO}_{2}$ at room temperature is $\alpha$-quartz (trigonal, space group $P 3_{2} 21$ or $P 3_{1} 21$ for right- or left-handed crystals, respectively). At elevated temperature it undergoes a reversible phase transition to $\beta$-quartz (hexagonal, space group $\mathrm{Pb}_{2} 22$ or $P 6_{4} 22$ ). The transition temperature is $573{ }^{\circ} \mathrm{C}(846 \mathrm{~K})$ at ambient pressure and is raised by $1{ }^{\circ} \mathrm{C}$ per 40 bar of pressure increase. There exist further polymorphs of silica, some of which will be considered in Sec. II.

Almost all silica found in nature at ambient temperature and pressure is $\alpha$-quartz. Quartz originally formed as $\alpha$-quartz will be referred to as low quartz, and quartz originally formed as $\beta$-quartz as high quartz. Characteristic differences between low and high quartz are found in the morphology and in the occurrence of twinning. Twins in which the principal axes of the two components are not parallel are rare in low quartz but occur frequently in high quartz; most frequent in high quartz are Esterel twins, ${ }^{1}$ the interface energy of which will be investigated by means of densityfunctional calculations.

\section{DENSITY-FUNCTIONAL CALCULATIONS}

The energy differences among the $\mathrm{SiO}_{2}$ polymorphs are quite small, on the order of or less than $0.1 \mathrm{eV}$ per $\mathrm{SiO}_{2}$ unit. ${ }^{2}$ This is small enough that the calculated energetic ordering may depend on the choice of the density functional and the variational basis sets in the calculation. Other computational details can be controlled well enough that no further issues about energetic ordering arise. As a representative sampling of density functionals we consider the local-density-functional approximation of the Perdew Wang correlation $^{3}$ (PWC) plus local exchange and the Perdew-BurkeErnzerhof (PBE) functional, ${ }^{4}$ which has an explicit dependence on density gradients. The PBE functional has recently been shown to be rather successful in predicting relative energies of a large number of molecular structures. ${ }^{5}$ It is widely used in solid state density-functional theory (DFT) calculations. The present calculations were done using the $\mathrm{DMol}^{3}$ code. ${ }^{6,7}$
Calculations were done with a double numerical with polarization (DNP) local orbital basis set. ${ }^{6}$ It consists of a double set of valence $s$ and $p$ functions, and a set of $d$-polarization functions arising from the product of one radial function and five $Y_{\ell, m}$ functions. As the basis set always includes the free atom orbital functions, the limit of (spin restricted, spherical) dissociated atoms is always attained exactly. The DNP basis set is found to consistently provide an excellent approximation for energies obtained with much larger and more computationally costly basis sets. DNP calculations run relatively fast and are thus useful for the large cells studied here. Larger basis sets of the $\mathrm{DMol}^{3}$ numerical type yield lower total energies and thus greater atomic dissociation energies than smaller basis sets. A triple valence set with double $d$ plus $f$ polarization (TNP) is such a basis set with significantly more variational flexibility und consequently lower energy with respect to dissociated atoms calculated numerically with high accuracy for the functional under consideration. Reference 5 shows that both DNP and TNP can reproduce experimental enthalpies very well for a wide class of molecules.

For the purpose of the present calculations a modified basis set of DNP type is considered $\left(\mathrm{DNP}_{\alpha}\right)$. This set is obtained by optimizing the variational basis functions so as to minimize the energy of an $\alpha$-quartz crystal. Table I shows experimental lattice parameters for four $\mathrm{SiO}_{2}$ structures. An important high-pressure, high-density polymorph and a simple low-pressure polymorph were taken for comparison with quartz.

The calculations using the PWC functional are total energy calculations for the experimental structures. The energy per $\mathrm{SiO}_{2}$ unit of $\alpha$-quartz is relative to a highly accurate numerical PWC solution for spin-unrestricted spherical atoms. The energies of the other structures are relative to $\alpha$-quartz with the same basis set. The calculations using the PBE functional are done with minimization of all structural and lattice parameters.

Table I shows that the PWC functional exaggerates bonding in the high-density structure of stishovite. As this persists for the larger variational basis set, it is clear that PWC erroneously identifies stishovite as the ground state at ambient pressure. 
TABLE I. Some $\mathrm{SiO}_{2}$ structures. Calculated density-functional total energies per $\mathrm{SiO}_{2}$ unit: $\alpha$-quartz relative to spin-unrestricted spherical atoms, energies of the other structures relative to $\alpha$-quartz. PWC calculations for experimental structure data, PBE calculations minimizing energy with respect to lattice constants, and Wyckoff position parameters of the atoms.

\begin{tabular}{|c|c|c|c|c|c|}
\hline \multicolumn{2}{|c|}{ Lattice constants } & $\begin{array}{c}\alpha \text {-quartz } \\
\text { Hexagonal }\end{array}$ & $\begin{array}{c}\beta \text {-quartz } \\
\text { Hexagonal }\end{array}$ & $\begin{array}{c}\text { High cristobalite } \\
\text { Cubic }\end{array}$ & $\begin{array}{l}\text { Stishovite } \\
\text { Tetragonal }\end{array}$ \\
\hline \multirow{2}{*}{\multicolumn{2}{|c|}{$\begin{array}{l}a(\AA) \\
c(\AA)\end{array}$}} & 4.910 & 4.998 & 7.166 & 4.176 \\
\hline & & 5.402 & 5.460 & & 2.665 \\
\hline Functional & Basis set & $(\mathrm{eV})$ & $(\mathrm{eV})$ & $(\mathrm{eV})$ & $(\mathrm{eV})$ \\
\hline PWC & TNP & -22.534 & +0.045 & +0.267 & -0.118 \\
\hline PWC & $\mathrm{DNP}_{\alpha}$ & -22.009 & +0.093 & +0.365 & -0.164 \\
\hline PBE & TNP & -20.006 & +0.074 & +0.051 & +0.565 \\
\hline PBE & $\mathrm{DNP}_{\alpha}$ & -19.453 & +0.043 & +0.098 & +0.409 \\
\hline
\end{tabular}

The calculations with the local density functional PWC relatively exaggerate the binding in stishovite. This happens for the TNP basis set as well as for the $\mathrm{DNP}_{\alpha}$ set. This misrepresentation of relative bonding is thus not coming from a basis set deficiency. It is rather a consequence of the PWC local-density-functional approximation. We expect that other local-density-functional approximations would show a similar imbalance.

The slightly more complicated functional PBE provides a more balanced approximation. For the experimental structural parameters $\alpha$-quartz has lowest energy. With lattice optimizations, the energy of high cristobalite is lowered more than the energies of other structures, but remains higher than the energy of $\alpha$-quartz, as should be. For the subsequent calculations we use the PBE functional with a DNP basis set optimized to minimize total energy for the experimental $\alpha$-quartz structure: $\mathrm{DNP}_{\alpha}$.

From $\Gamma$ point vibrational calculations we estimate that $\beta$-quartz has a $0.45 \mathrm{kcal} / \mathrm{mol}$ lower zero-point vibrational energy and a $1.1 \mathrm{cal} /(\mathrm{mol} \mathrm{K})$ higher vibrational entropy than $\alpha$-quartz. The observation of an $\alpha-\beta$ phase transition at $846 \mathrm{~K}$ suggests then that the total energy of $\beta$-quartz is about $0.06 \mathrm{eV}$ higher than for $\alpha$-quartz, in semiquantitative agreement with our DFT results.

The calculated lattice constants differ from the experimental ones as a consequence of the approximations inherent to the functional and, to a lesser degree, due to the variational basis set used here and to further numerical approximations (see Table II).

Distorsions at the twin interface raise interest in elastic properties. Elastic stiffness constants were calculated using least-squares fitting for a set of calculations with small strains (see Table III).

TABLE II. Lattice parameters at PBE energy minimum relative to experimental ones.

\begin{tabular}{ccccc} 
Lattice parameter & $\alpha$-quartz & $\beta$-quartz & High cristobalite & Stishovite \\
\hline$a$ & $+0.8 \%$ & $+2.2 \%$ & $+4.4 \%$ & $+1.4 \%$ \\
$c$ & $+1.0 \%$ & $+2.4 \%$ & & $+1.7 \%$ \\
\hline \hline
\end{tabular}

The calculated diagonal stiffness constants (including the shear constants $c_{44}$ and $c_{66}$ ) appear slightly too soft as compared to experiment.

\section{ESTEREL TWIN MODELS WITH PERIODIC BOUNDARY CONDITIONS}

\section{A. Esterel twinning}

We start our discussion of twins in $\alpha$ - or $\beta$-quartz using the conventional hexagonal crystal coordinate system with basis vectors $\mathbf{a}, \mathbf{b}, \mathbf{c}$ satisfying $|\mathbf{a}|=|\mathbf{b}|=a,|\mathbf{c}|=c$, and $\alpha=\beta$ $=90^{\circ}, \gamma=120^{\circ}$, where $a$ and $c$ take the values at the PBE energy minimum, as indicated in Table II. Notice that $\mathbf{a}, \mathbf{b}$, and $\mathbf{c}$ span a primitive cell of the quartz lattice, the volume of which is $\frac{1}{2} \sqrt{3} \mathbf{a}^{2} \mathbf{c}$. The most common twin in high-quartz is the Esterel twin. ${ }^{1,9}$ From a macroscopic point of view, the two individuals of an Esterel twin are related by a mirror reflection in the plane $(0 \overline{1} 11)$ if the two individuals are of opposite hand and by a $180^{\circ}$ rotation about the axis [01 $\left.\overline{1} 2\right]$ $=[122]$ if the two individuals are of the same hand. Notice that the axis $[01 \overline{1} 2]$ is parallel to the twin plane $(0 \overline{1} 11)$. The composition plane is parallel to $(0 \overline{1} 11)$ in most cases, but also composition planes perpendicular to $(0 \overline{1} 11)$ have been observed.

Twins with twin plane $(0 \overline{1} 11)$ are much less common in low quartz, where they are known as Reichenstein-

TABLE III. Elastic stiffness constants of $\alpha$-quartz in GPa. $c_{66}$ $=\left(c_{11}-c_{12}\right) / 2$.

\begin{tabular}{ccc}
\hline \hline & Experiment (Ref. 8) & PBE DNP $_{\alpha}$ \\
\hline$c_{11}$ & $85.0-87.3$ & 80.1 \\
$c_{33}$ & $105.5-107.7$ & 103.7 \\
$c_{44}$ & $57.2-59.7$ & 55.0 \\
$c_{12}$ & $4.8-9.9$ & 9.9 \\
$c_{13}$ & $10.5-15.1$ & 16.0 \\
$c_{14}$ & -16.8 to -18.3 & -13.0 \\
\hline \hline
\end{tabular}




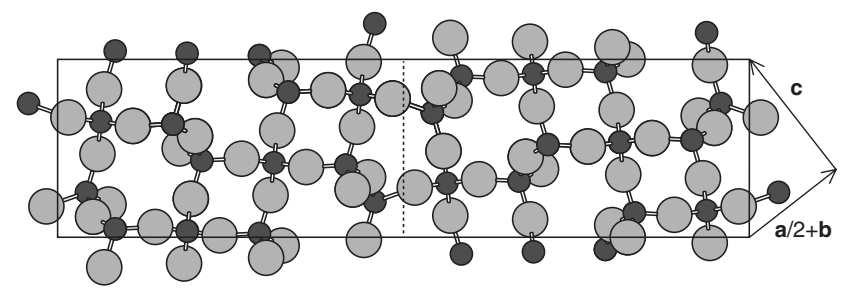

FIG. 1. Model of Esterel twin with four primitive cell layers with unrelaxed bulk positions in each component. View along the symmetry translation vector $\mathbf{a}$ in the composition plane. $\mathrm{O}$ atoms are shown as large gray dots, $\mathrm{Si}$ atoms as small black dots. Supplementary atoms have been added outside the cell to show all bonds that emerge from the cell.

Grieserntal twins. ${ }^{1}$ For simplicity, we shall speak of Esterel twins in both cases.

A model of the arrangement of the atoms in an Esterel twin boundary with composition plane parallel to the twin plane has been proposed in Fig. 5 of Ref. 10, where the mirror reflection in the composition plane passes through $\mathrm{O}$ ions. This model seems unrealistic to us because it has $\mathrm{Si}$ ions at close distance on both sides of the twin boundary. The $\mathrm{O}$ ions necessary for charge neutrality at the interface are missing because there is no space for them.

In the following subsections we first construct Esterel twins from the unrelaxed bulk quartz lattice. In Sec. IV we study the relaxation of these twin models with DFT, while keeping the composition plane fixed.

\section{B. Esterel reflection twins}

A more realistic model of an Esterel reflection twin is proposed in Fig. 1. The vectors marked $\mathbf{c}$ and $\frac{1}{2} \mathbf{a}+\mathbf{b}+\mathbf{c}$ refer to the orientation of the component on the left-hand side. The composition plane contains a, which is normal to the plane of the drawing, and $\frac{1}{2} \mathbf{a}+\mathbf{b}+\mathbf{c}$. Figure 1 shows the starting point for one of the present calculations. It has unrelaxed $\beta$-quartz positions in each twin component. The figure shows that the component on the right-hand side was obtained not simply by a mirror reflection in the composition plane at the center of the figure, but by a glide reflection. The glide vector is $\frac{1}{2}(\mathbf{a}+\mathbf{b}+\mathbf{c})$, which has a component $\frac{1}{2}\left(\frac{1}{2} \mathbf{a}+\mathbf{b}+\mathbf{c}\right)$ in the plane of the drawing and a component $\frac{1}{4}$ a perpendicular to it. The position of the glide plane was chosen such that the starting twin structure has the same density as the bulk.

TABLE IV. Distances between neighboring ions for our model of the Esterel reflection twin in $\beta$-quartz with lattice constants as indicated in Table II. The glide vector is $(1 / 2)(\mathbf{a}+\mathbf{b}+\mathbf{c})$ for solution $1,(1 / 2)(\mathbf{b}+\mathbf{c})$ for solution 2 .

\begin{tabular}{cccc}
\hline \hline & & \multicolumn{2}{c}{ Across twin boundary } \\
\cline { 3 - 4 } Distance $(\AA)$ & Bulk & Solution 1 & Solution 2 \\
\hline Smallest Si-O distance & 1.628 & 1.957 & 2.312 \\
Smallest O-O distance & 2.602 & 1.936 & 2.504 \\
\hline \hline
\end{tabular}

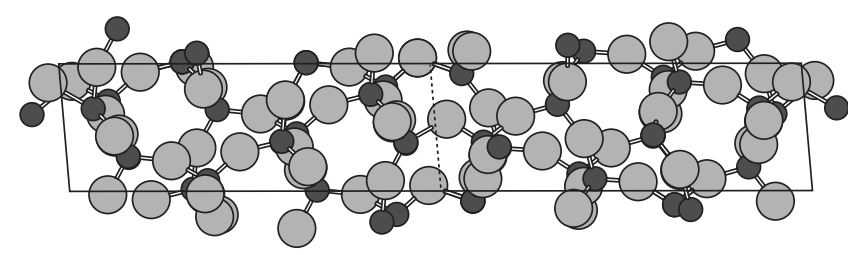

FIG. 2. Model of Esterel reflection twin with four primitive cell layers in each component. View along the symmetry translation $\mathbf{p}_{2}$ in the composition plane.

Periodic boundary conditions are needed for DFT calculations, i.e., we have to consider a periodic arrangement of twin boundaries, a periodic polysynthetic twin. Figure 1 shows composition planes at its left and right ends and at the center (marked by vertical lines); the vector $4 \mathbf{c}$ connects neighboring composition planes, i.e., each twin lamella has a thickness of four single-crystal periods normal to the composition planes. Each composition plane is a glide-reflection plane of the polysynthetic twin with glide vector $\frac{1}{2}(\mathbf{a}+\mathbf{b}$ $+\mathbf{c})$. The polysynthetic twin has an all-side centered orthorhombic cell $(o F)$ with a conventional basis consisting of the three mutually orthogonal vectors $\mathbf{v}_{1}=\mathbf{a}, \mathbf{v}_{2}=\mathbf{a}+2 \mathbf{b}+2 \mathbf{c}, \mathbf{v}_{3}$ $=16 \mathbf{c}-\left[32 c^{2} /\left(3 a^{2}+4 c^{2}\right)\right](\mathbf{a}+2 \mathbf{b}+2 \mathbf{c}) ;$ the space group is $F d d 2$ (no. 43). If the thickness of a twin lamella is $n$ single crystal periods, the space group and $\mathbf{v}_{1}$ and $\mathbf{v}_{2}$ remain the same, whereas $\mathbf{v}_{3}$ becomes $\mathbf{v}_{3}=4 n \mathbf{c}-\left[8 n c^{2} /\left(3 a^{2}+4 c^{2}\right)\right](\mathbf{a}$ $+2 \mathbf{b}+2 \mathbf{c})$. The volume of the cell spanned by $\mathbf{v}_{1}, \mathbf{v}_{2}$, and $\mathbf{v}_{3}$, i.e., the volume of a conventional cell of the polysynthetic twin, is $4 n \sqrt{3} \mathbf{a}^{2} \mathbf{c}$, the volume of a primitive cell therefore $n \sqrt{3} \mathbf{a}^{2} \mathbf{c}$, i.e., $2 n$ times the volume of a primitive cell of the quartz lattice.

Table IV gives the distances between neighboring ions in the bulk and across the composition plane for our model of the Esterel reflection twin, where the glide vector has been chosen as $\frac{1}{2}(\mathbf{a}+\mathbf{b}+\mathbf{c})$ and for an alternative model with glide vector $\frac{1}{2}(\mathbf{b}+\mathbf{c})$. The component of the glide vector perpendicular to the plane of the drawing of Fig. 1 is $+\frac{1}{2} \mathbf{a}$ in the first case, $-\frac{1}{2} \mathbf{a}$ in the second.

Table IV shows that even for solution 2 the distance between the closest $\mathrm{O}$ ions across the twin boundary is smaller than in the bulk. We conclude that, as expected, the density in the boundary will in reality be less than in the bulk as a consequence of $\mathrm{O}-\mathrm{O}$ repulsion.

Figure 1 showed our model of an $n=4$ polysynthetic Esterel reflection twin projected along a. Solution 2 looks the

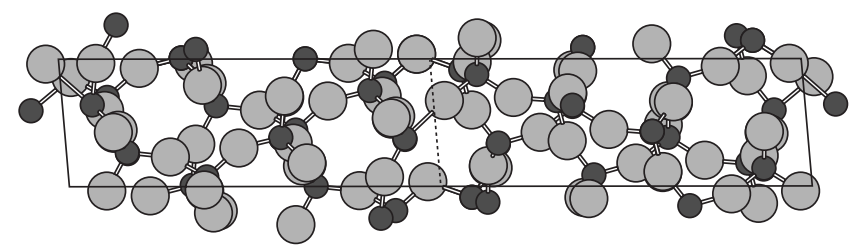

FIG. 3. Model of Esterel rotation twin with four primitive cell layers in each component. View along the vector $\mathbf{a}+\mathbf{b}+\mathbf{c}$ in the composition plane. The glide vector of the $180^{\circ}$ screw rotation connecting neighboring components is $\frac{1}{2}(\mathbf{a}+\mathbf{b}+\mathbf{c})$ at all twin boundaries, leading to an all-side centered orthorhombic cell. 


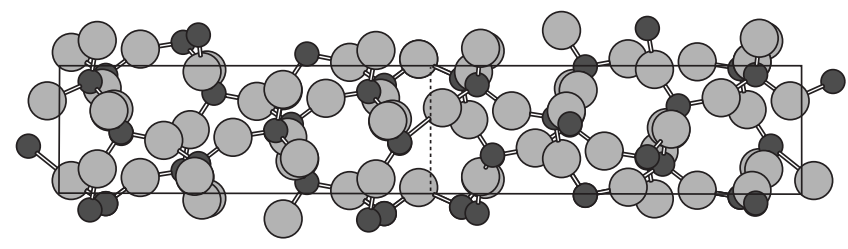

FIG. 4. Model of Esterel rotation twin with four primitive cell layers in each component. View along the vector $\mathbf{a}+\mathbf{b}+\mathbf{c}$ in the composition plane. The glide vector of the $180^{\circ}$ screw rotation is $\frac{1}{2}(\mathbf{a}+\mathbf{b}+\mathbf{c})$ at the central twin boundary and $\frac{1}{2}(\mathbf{b}+\mathbf{c})$ at the two outer twin boundaries, leading to a one-side centered orthorhombic cell. [Notice that the two glide vectors $\frac{1}{2}(\mathbf{a}+\mathbf{b}+\mathbf{c})$ and $\frac{1}{2}(\mathbf{b}+\mathbf{c})$ give rise to twin boundaries with the same local symmetry, differing only by a translation.]

same as solution 1 in this projection. In the following we shall restrict our attention to solution 1. A primitive cell is spanned by $\mathbf{p}_{1}=\mathbf{v}_{1}=\mathbf{a}, \quad \mathbf{p}_{2}=\frac{1}{2}\left(\mathbf{v}_{1}+\mathbf{v}_{2}\right)=\mathbf{a}+\mathbf{b}+\mathbf{c}, \quad \mathbf{p}_{3}=\frac{1}{2}\left(\mathbf{v}_{1}\right.$ $+\mathbf{v}_{3}$ ). Notice that $\mathbf{p}_{1}$ and $\mathbf{p}_{2}$ lie in the composition plane. Figure 1 can now be interpreted as normal projection of this primitive cell on a plane perpendicular to $\mathbf{p}_{1}$. Figure 2 shows this cell in normal projection on a plane perpendicular to $\mathbf{p}_{2}$.

The model shown in Figs. 1 and 2 will serve as starting point for our density-functional calculations of the Esterel reflection twin, in which the symmetry translations $\mathbf{v}_{1}$ and $\mathbf{v}_{2}$ in the composition plane will be left fixed, whereas $\mathbf{v}_{3}$ will be allowed to change its length.

\section{Esterel rotation twins}

In our models of Esterel rotation twins the orientation of the composition plane is the same as for reflection twins and its position again such that twinned and untwinned crystals have the same density. The two components are related by a $180^{\circ}$ screw rotation about an axis in the composition plane parallel to $\frac{1}{2} \mathbf{a}+\mathbf{b}+\mathbf{c}$ with glide vector $\frac{1}{2}(\mathbf{a}+\mathbf{b}+\mathbf{c})$. This glide vector has a component $\frac{1}{2}\left(\frac{1}{2} \mathbf{a}+\mathbf{b}+\mathbf{c}\right)$ parallel to the screw axis and a component $\frac{1}{2}$ a perpendicular to it.

The periodic boundary conditions needed for DFT calculations can be obtained in two different ways: (1) the component of the glide vector perpendicular to the screw axis is $\frac{1}{2} \mathbf{a}$ in every twin plane or (2) it changes sign between neighboring twin planes, in which case the total glide vector alternates between $\frac{1}{2}(\mathbf{a}+\mathbf{b}+\mathbf{c})$ (as in solution 1 for the reflection twin) and $\frac{1}{2}(\mathbf{b}+\mathbf{c})($ as in solution 2 for the reflection twin).

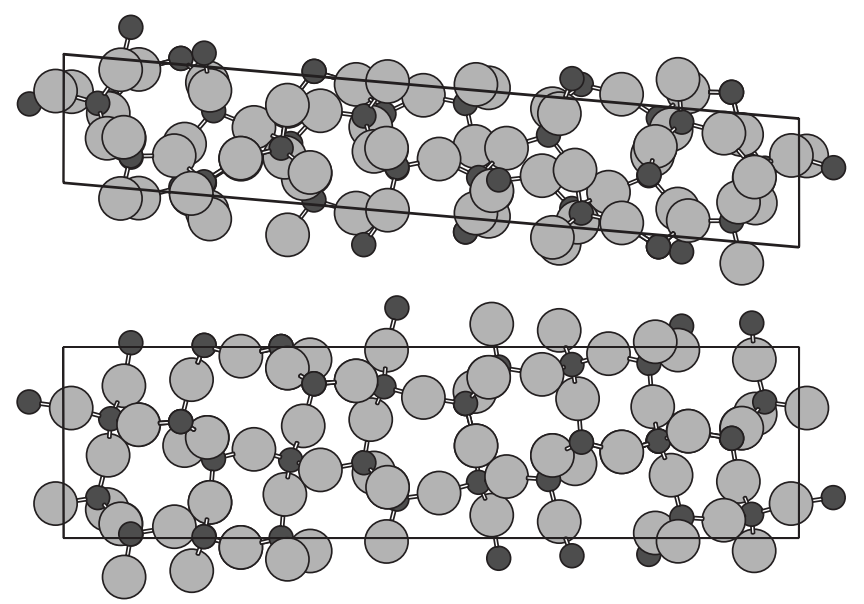

FIG. 5. Relaxed reflection twin, viewed along $\mathbf{a}+\mathbf{b}+\mathbf{c}$ (above) and along a (below).

Both glide vectors lead to shortest $\mathrm{Si}-\mathrm{O}$ and O-O distances across the twin boundary that alternate between the values for the reflection twin solutions 1 and 2 .

In case 1 the polysynthetic rotation twin has the same periodicity as the reflection twin, i.e., a conventional all-side centered cell spanned by the three mutually orthogonal vectors $\mathbf{v}_{1}, \mathbf{v}_{2}, \mathbf{v}_{3}$, and a primitive cell spanned by $\mathbf{p}_{1}, \mathbf{p}_{2}, \mathbf{p}_{3}$. This cell is shown in Fig. 3 .

In case 2 the polysynthetic rotation twin has a conventional one-side centered cell spanned by the three mutually orthogonal vectors $\mathbf{v}_{1}, \mathbf{v}_{2}, \frac{1}{2} \mathbf{v}_{3}$ and a primitive cell spanned by $\mathbf{p}_{1}=\mathbf{v}_{1}=\mathbf{a}, \mathbf{p}_{2}=\frac{1}{2}\left(\mathbf{v}_{1}+\mathbf{v}_{2}\right)=\mathbf{a}+\mathbf{b}+\mathbf{c}$, and by $\frac{1}{2} \mathbf{v}_{3}$. This cell is shown in Fig. 4.

Viewed along a, the structures shown in Figs. 3 and 4 look as in Fig. 1. Whereas our model of the periodic polysynthetic reflection twin had the space group symmetry $F d d 2$, the models of rotation twins have only monoclinic symmetry, $C 2$ (no. 5) in the case of Fig. 3 and $P 2_{1}$ (no. 4) in the case of Fig. 4. [A model of a periodic polysynthetic rotation twin with orthorhombic symmetry $C 222_{1}$ (no. 20) can be constructed but has ions of the same charge next to each other across the composition planes.] The symmetry direction of $C 2$ is along $\mathbf{v}_{1}$, the symmetry direction of $P 2_{1}$ along $\mathbf{v}_{3}$.

Figures 1-4 have been constructed from the $\beta$-quartz structure. The symmetry of the polysynthetic twin is low, so that the corresponding polysynthetic twins for $\alpha$-quartz have the same space group. This fact has an immediate conse-

TABLE V. Distances between neighboring ions for the Esterel reflection twin in $\beta$-quartz with lattice constants as indicated in Table II.

\begin{tabular}{|c|c|c|c|c|c|}
\hline \multirow[b]{3}{*}{ Distance $(\AA)$} & \multicolumn{2}{|r|}{ Unrelaxed } & \multicolumn{3}{|c|}{ Relaxed } \\
\hline & \multirow[b]{2}{*}{ Bulk } & \multirow[b]{2}{*}{ Across twin boundary } & \multicolumn{2}{|c|}{ Bulk } & \multirow[b]{2}{*}{ Across twin boundary } \\
\hline & & & Min. & Max. & \\
\hline Smallest Si-O distance & 1.628 & 1.957 & 1.618 & 1.646 & 1.638 \\
\hline Smallest O-O distance & 2.602 & 1.936 & 2.594 & 2.644 & 2.606 \\
\hline
\end{tabular}


TABLE VI. Energies and displacements per interface. $E_{\mathrm{twin}}$ is the energy per area of a primitive cell, stretch gives the extension normal to the interface, and shear across the interface is parallel to $\mathbf{v}_{2}$.

\begin{tabular}{|c|c|c|c|c|c|c|c|}
\hline \multirow[b]{2}{*}{ Twin type } & \multirow[b]{2}{*}{$\begin{array}{l}\text { Number } \\
\text { of layers }\end{array}$} & \multicolumn{3}{|c|}{$\beta$-quartz } & \multicolumn{3}{|c|}{$\alpha$-quartz } \\
\hline & & $\begin{array}{l}E_{\text {twin }} \\
(\mathrm{eV})\end{array}$ & $\begin{array}{l}\text { Stretch } \\
(\mathrm{pm})\end{array}$ & $\begin{array}{l}\text { Shear } \\
(\mathrm{pm})\end{array}$ & $\begin{array}{l}E_{\text {twin }} \\
(\mathrm{eV})\end{array}$ & $\begin{array}{l}\text { Stretch } \\
(\mathrm{pm})\end{array}$ & $\begin{array}{l}\text { Shear } \\
(\mathrm{pm})\end{array}$ \\
\hline Reflection & 1 & 0.14 & 10 & & 0.25 & 11 & \\
\hline \multirow[t]{6}{*}{$F d d 2$} & 2 & 0.51 & 17 & & 0.62 & 20 & \\
\hline & 3 & 0.75 & 21 & & 0.70 & 23 & \\
\hline & 4 & 0.59 & 21 & & 0.64 & 22 & \\
\hline & 5 & 0.69 & 23 & & 0.98 & 39 & \\
\hline & 6 & 0.68 & 25 & & 0.95 & 41 & \\
\hline & 7 & 0.74 & 38 & & 1.02 & 41 & \\
\hline Rotation & 1 & 0.56 & 2 & 2 & 0.49 & 6 & 2 \\
\hline$C 2$ & 2 & 0.76 & -8 & -1 & 0.82 & 2 & -5 \\
\hline monoclinic & 3 & 0.99 & -6 & -13 & 0.89 & 4 & -17 \\
\hline \multirow[t]{2}{*}{$\operatorname{axis} \mathbf{v}_{1}$} & 4 & 0.84 & 4 & 7 & 0.79 & 3 & -11 \\
\hline & 5 & 0.99 & -1 & -10 & 0.89 & 7 & -14 \\
\hline Rotation & 1 & 1.34 & -6 & & 1.19 & -4 & \\
\hline$P 2_{1}$ & 2 & 0.91 & 2 & & 0.78 & 1 & \\
\hline monoclinic & 3 & 0.94 & -4 & & 0.86 & 0 & \\
\hline \multirow[t]{2}{*}{$\operatorname{axis} \mathbf{v}_{3}$} & 4 & 0.93 & -4 & & 0.99 & 22 & \\
\hline & 5 & 0.95 & -3 & & 1.02 & 17 & \\
\hline
\end{tabular}

quence for hypothetical calculations in the limit of many layers in each twin component. If we assume the ideal twin interface as a dislocation-free, strained structure, the translation vectors in the composition plane are fixed at the $\beta$-quartz values. But as the low-temperature ground state is $\alpha$-quartz, the bulk, i.e., the middle part of the thick twin components, must converge to uniaxially strained $\alpha$-quartz in such (not feasible) calculations. The uniaxial strain arises from the fact that the two-dimensional interface lattice parameters are kept fixed at the $\beta$-quartz structure.

Corresponding models have been calculated also for other
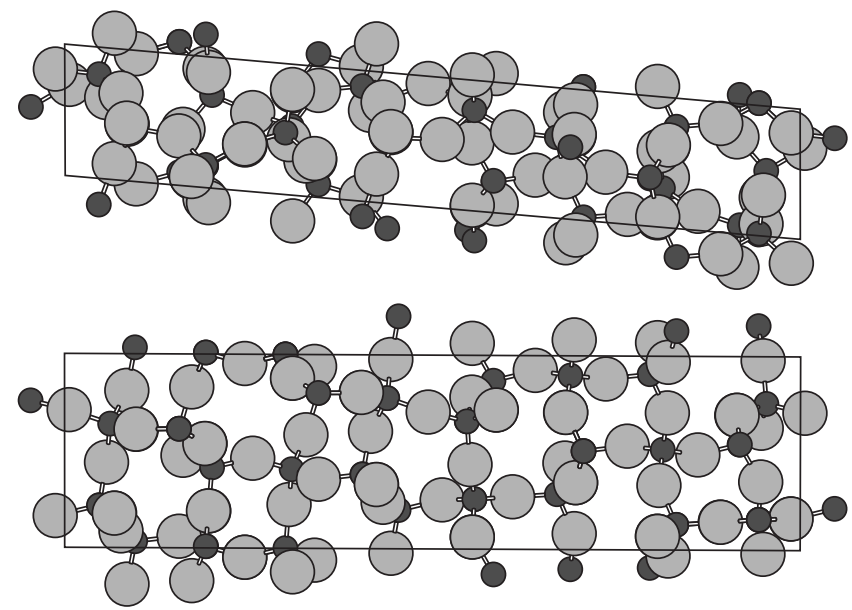

FIG. 6. Relaxed rotation twin of Fig. 3, viewed along $\mathbf{a}+\mathbf{b}+\mathbf{c}$ (above) and along a (below). thickness values of the twin components and also for $\alpha$-quartz.

\section{DFT RELAXATION OF THE TWIN INTERFACE}

The models illustrated in Figs. 1-4 serve as starting points for our density-functional calculations of the Esterel twins. The symmetry translations $\mathbf{v}_{1}$ and $\mathbf{v}_{2}$ in the composition plane will be left fixed. In the case shown in Fig. 3, the monoclinic axis is $\mathbf{v}_{1}$. It follows that $\mathbf{v}_{3}$ is allowed to change its length and the angle that it makes with $\mathbf{v}_{2}$. The cell may

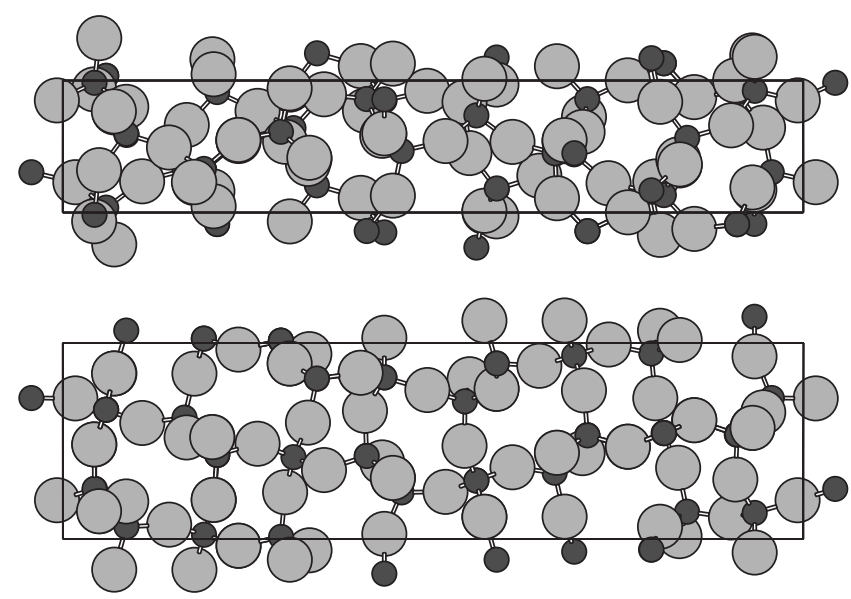

FIG. 7. Relaxed rotation twin of Fig. 4, viewed along $\mathbf{a}+\mathbf{b}+\mathbf{c}$ (above) and along a (below). 
then become monoclinic. In the case shown in Fig. 4 the monoclinic axis is $\mathbf{v}_{3}$, from which it follows that $\mathbf{v}_{3}$ can change only its length. The metric of the cell then remains orthorhombic.

The relaxation of all parameters except the lattice parameters in the composition plane leads to an atomic rearrangement which is shown in Fig. 5 for the reflection twin. The vector $\mathbf{v}_{3}$ being normal to the composition plane, its length remains free for relaxation.

The relaxation brings the $\mathrm{Si}-\mathrm{O}$ bond distance and the smallest $\mathrm{O}-\mathrm{O}$ distance back very close to the bulk values (Table V). It is interesting to note that the extremal values of the bond length for the relaxed structure do not occur across the composition plane.

The energies of the relaxed twin models can be compared to the bulk energies to extract the interfacial energy of twinning:

$$
E_{t}\left(n_{\text {layer }}\right)=n_{\text {layer }} E_{\alpha}^{1}+2 E_{\text {twin }},
$$

where $E_{\alpha}^{1}$ is the relevant bulk energy per cell. In the case of $\alpha$-quartz interface geometry, this is just the bulk energy of $\alpha$-quartz. In the case of $\beta$-quartz interface geometry, $E_{\alpha}^{1}$ is the bulk energy of uniaxially strained $\alpha$-quartz matching the constrained geometry of the composition plane.

It is possible to do low-temperature bulk $\beta$-quartz calculations by maintaining the high symmetry of the $\beta$ phase. Because of the low symmetry of the twin model, this approach is not possible here. A proper calculation of the interface enthalpy in the $\beta$ phase requires in principle vibrational calculations for the entropy term in the enthalpy. This is beyond the scope of the present work. We know that the different vibrational entropy contributions let the enthalpies as a function of temperature cross at the phase transition. The present calculations lead to quite similar estimations of the interfacial energies of $\alpha$ - and $\beta$-quartz, as shown in Table VI. The vibrational calculation would be expected to show softened vibrations due to the stretched bonds at the interface, which in turn lead to a slight lowering of the interface enthalpy at elevated temperature. The Esterel reflection twin has clearly lower energy than the two rotation twins. As its nucleation energy must be connected to this interfacial energy, it is evident that (even at $\beta$ phase temperatures) spontaneous thermal formation of this defect will occur only rarely. Indeed, occurrence is typically much less than one such interface per specimen. The present calculations suggest that occurrence of an Esterel rotation twin interface is even more rare.

The next figures show the relaxed structures for the rotation twins with symmetry $C 2$ (Fig. 6) and symmetry $P 2_{1}$ (Fig. 7).

\section{DISCUSSION}

The twin energies given in Table VI do not converge versus component thickness. Relaxation takes place deep into the twin components. The present calculations suggest that for the $\beta$-quartz reflection twin the interfacial energy is $0.7 \mathrm{eV}$ per surface cell, but convergence with thickness is probably only within $\pm 0.2 \mathrm{eV}$. It is interesting to note that the low-thickness calculations all give relatively low twin energies for the reflection twin and the rotation twin with symmetry axis $\mathbf{v}_{1}$.

The interface energies of the polysynthetic twins studied here suggest an even-odd alternation with the number of primitive units in each twin component. The occurrence of this appears to be a consequence of having stiff $\mathrm{SiO}_{4}$ tetrahedra connected flexibly at the vertices. For thicker twin components than studied here the oscillation should die out. The oscillation is irrelevant for natural specimens, which typically contain a single twin interface.

\section{SUMMARY}

We have presented models for Esterel twin interfaces with mirror and rotation symmetry that satisfy stoichiometry at the composition plane. The starting points are unrelaxed models constructed from bulk crystal and keeping the bulk volume per formula unit. the present unrelaxed models also maintain the fourfold coordination of all $\mathrm{Si}$ atoms and the twofold coordination of the $\mathrm{O}$ atoms, albeit with significant variation of bond length and bond angles at the composition plane. More realistic atomic positions were sought with density-functional calculations. Relaxation energies are quite significant, as the initial unrelaxed interface energy is about $15 \mathrm{eV}$ above the relaxed energy for our cells containing two twin interfaces. After relaxation, bond length and bond angles are close to bulk quartz values. The energetics from DFT say that the mirror symmetry twinning has the lowest interface energy among the models investigated here.
${ }^{1}$ C. Frondel, The System of Mineralogy (Wiley, New York, 1962), Vol. III.

${ }^{2}$ http://webbook.nist.gov/

${ }^{3}$ J. P. Perdew and Y. Wang, Phys. Rev. B 45, 13244 (1992).

${ }^{4}$ J. P. Perdew, K. Burke, and M. Ernzerhof, Phys. Rev. Lett. 77, 3865 (1996).

${ }^{5}$ B. Delley, J. Phys. Chem. A 110, 13632 (2006).

${ }^{6}$ B. Delley, J. Chem. Phys. 92, 508 (1990).
${ }^{7}$ B. Delley, J. Chem. Phys. 113, 7756 (2000).

${ }^{8}$ G. Simmons and H. Wang, Single Crystal Elastic Constants, 2nd ed. (MIT Press, Cambridge, MA, 1971).

${ }^{9}$ J. Drugman, Miner. Mag. 21, 366 (1972).

${ }^{10}$ A. C. McLaren, in Mineral and Rock Deformation: Laboratory Studies, edited by B. E. Hobbs and H. C. Heard, Geophysical Monographs Vol. 36 (American Geophysical Union, Washington D.C., 1986), pp. 233-245. 